International Mathematical Forum, 2, 2007, no. 65, 3219 - 3231

\title{
Grothendieck Group of an Equivariant Derived Category
}

\author{
Mladen Božičević \\ Department of Geotechnical Engineering, University of Zagreb \\ Hallerova 7, 42000 Varaždin, Croatia \\ mladen.bozicevic@gmail.com
}

\begin{abstract}
Let $G$ be a topological group acting on a topological space $X$. Let $k$ be a field. Denote by $S h_{G}(X)$ the category of equivariant sheaves of $k$-vector spaces on $X$, and by $D_{G}^{b}(X)$ the bounded equivariant derived category. We show that the Grothendieck groups of the categories $S h_{G}(X)$ and $D_{G}^{b}(X)$ are naturally isomorphic.
\end{abstract}

Mathematics Subject Classification: 18E30, 18F20, 54B40, 55N91

Keywords: Equivariant derived category, Grothendieck group, triangulated category, t-structure

\section{Introduction}

Let $G$ be a topological group acting continuously on a topological space $X$. We denote by $S h_{G}(X)$ the category of $G$-equivariant sheaves of vector spaces over a fixed field. Let $f: X \longrightarrow Y$ be a $G$-equivariant map. In order to extend the formalism of six operations $f^{*}, R f_{*}, R f_{!}, f^{!}, R H o m$ and $\otimes^{L}$ from the derived categories to the equivariant setting, Bernstein and Lunts introduced in [1] the eqivariant derived categories $D_{G}^{b}(X)$ and $D_{G}^{+}(X)$. We recall briefly the definition of $D_{G}^{b}(X)$. Let $E G$ be the the universal bundle of $G$. Consider $P=X \times E G$ as a $G$-space for the diagonal action of $G$. Denote by $p: P \longrightarrow X$ the projection, and by $q: P \longrightarrow \bar{P}=X \times{ }_{G} E G$ the quotient map. Then the objects of $D_{G}^{b}(X)$ consist of triples $\mathcal{F}=\left(\mathcal{F}_{X}, \overline{\mathcal{F}}, \alpha\right)$, where $\mathcal{F}_{X} \in O b\left(D^{b}(X)\right)$, $\overline{\mathcal{F}} \in O b\left(D^{b}(\bar{P})\right)$, and $\alpha: p^{*}\left(\mathcal{F}_{X}\right) \simeq q^{*}(\overline{\mathcal{F}})$ is an isomorphism in $D^{b}(P)$. A morphism $\beta: \mathcal{F} \longrightarrow \mathcal{F}^{\prime}$ is a pair of morphisms $\beta_{X}: \mathcal{F}_{X} \longrightarrow \mathcal{F}_{X}^{\prime}$ and $\bar{\beta}: \overline{\mathcal{F}} \longrightarrow$ $\overline{\mathcal{F}}^{\prime}$ satisfying the compatibility condition $q^{*}(\bar{\beta}) \alpha=\alpha^{\prime} p^{*}\left(\beta_{X}\right)$. The pull-back of distinguished triangles and standard $t$-structure from $D^{b}(\bar{P})$ by the restriction functor $\mathcal{F} \longrightarrow \overline{\mathcal{F}}$, determines the structure of a triangulated $t$-category on 
$D_{G}^{b}(X)$. It is known that the heart of the $t$-structure on $D_{G}^{b}(X)$ is naturally isomorphic to the category $S h_{G}(X)$. The proof of this fact in [1] follows from the simplicial description of the category $D_{G}^{b}(X)$, which is based on the results of Deligne about simplicial topological spaces [3].

In the present paper we propose an alternative approach to the computation of the heart of the $t$-structure of $D_{G}^{b}(X)$. In addition, we also compare the Grothendieck groups of the categories $S h_{G}(X)$ and $D_{G}^{b}(X)$, and obtain that they are naturally isomorphic. This is deduced from a general result about the Grothendieck group of a triangulated category with a non-degenerate and bounded $t$-structure. In the special case when a Lie group acts analytically with finitely many orbits on a manifold $X$ our results could be deduced (for example) from [7, Lem.6.4].

\section{Review of $t$-structures}

Given a category $\mathcal{T}$ we denote by $O b(\mathcal{T})$ the objects of $\mathcal{T}$. Suppose that $\mathcal{T}$ is an additive category. A translation functor on $\mathcal{T}$ is an additive automorphism $T: \mathcal{T} \longrightarrow \mathcal{T}$. For $k \in \mathbb{Z}$ we write $T^{k}(A)=A[k], A \in O b(\mathcal{T})$. A triangle in $\mathcal{T}$ is a sequence of morphisms

$$
A \longrightarrow B \longrightarrow C \longrightarrow A[1]
$$

Sometimes we shall denote it simply by $(A, B, C)$. An additive category $\mathcal{T}$ is called a triangulated category if it is equipped with a translation functor and a family of triangles, called distinguished triangles, which satisfy certain natural axioms [4, IV.1.1]. We shall refer to these axioms as (TR1)-(TR4).

Let $\mathcal{T}$ be a triangulated category, and $\mathcal{D}$ a full subcategory stable for $T$. We say that $\mathcal{D}$ is closed under extensions if for any triangle $(A, B, C)$ in $\mathcal{T}$, where $A, C \in O b(\mathcal{D})$, we have $B \in O b(\mathcal{D})$. The proof of the following lemma is straightforward.

Lemma 1.1 Let $\mathcal{T}$ be a triangulated category, and let $\mathcal{D}$ be a full subcategory. Assume $\mathcal{D}$ has the following properties:

1. $\mathcal{D}$ is an additive subcategory;

2. $\mathcal{D}$ is stable for $T$;

3. $\mathcal{D}$ is closed under extensions.

Define distinguished triangles in $\mathcal{D}$ as distinguished triangles $(A, B, C)$ in $\mathcal{T}$ such that $A, B, C \in O b(\mathcal{D})$. Then $\mathcal{D}$ has a structure of triangulated category. We shall say that with this structure $\mathcal{D}$ is a triangulated subcategory of $\mathcal{T}$. 
Next we recall the definition of $t$-structure on a triangulated category [2]. Let $\mathcal{D}$ be a triangulated category, and $\left(\mathcal{D}^{\leq 0}, \mathcal{D}^{\geq 0}\right)$ a pair of full subcategories. Put $\mathcal{D}^{\leq n}=\mathcal{D}^{\leq 0}[-n]$ and $\mathcal{D}^{\geq n}=\mathcal{D}^{\geq 0}[-n]$. Then $\left(\mathcal{D}^{\leq 0}, \mathcal{D}^{\geq 0}\right)$ is said to be a $t$-structure on $\mathcal{D}$ if it satisfies the following conditions:

1. $\mathcal{D}^{\leq-1} \subset \mathcal{D}^{\leq 0}$ and $\mathcal{D}^{\geq 1} \subset \mathcal{D}^{\geq 0}$;

2. If $A \in O b\left(\mathcal{D}^{\leq 0}\right)$ and $B \in O b\left(\mathcal{D}^{\geq 1}\right)$, then $\operatorname{Hom}_{\mathcal{D}}(A, B)=0$;

3. For any $A \in O b(\mathcal{D})$, there exists a distinguished triangle $\left(A_{0}, A, A_{1}\right)$ in $\mathcal{D}$ with $A_{0} \in O b\left(\mathcal{D}^{\leq 0}\right)$ and $A_{1} \in O b\left(\mathcal{D}^{\geq 1}\right)$.

The full subcategory $\mathcal{C}=\mathcal{D}^{\leq 0} \cap \mathcal{D}^{\geq 0}$ is called the heart of the $t$-structure. The inclusion $\mathcal{D}^{\leq n} \longrightarrow \mathcal{D}$ has a right adjoint functor $\tau_{\leq n}: \mathcal{D} \longrightarrow \mathcal{D}^{\leq n}$. Similarly, the inclusion $\mathcal{D}^{\geq n} \longrightarrow \mathcal{D}$ has a left adjoint functor $\tau_{\geq n}: \mathcal{D} \longrightarrow \mathcal{D}^{\geq n}$.

Recall that for any $A \in O b(\mathcal{D})$ there exists a unique morphism $d: \tau_{\geq n+1}(A)$ $\longrightarrow \tau_{\leq n}(A)[1]$, which together with adjointness morphisms defines a distinguished triangle

$$
\tau_{\leq n}(A) \stackrel{i}{\longrightarrow} A \stackrel{j}{\longrightarrow} \tau_{\geq n+1}(A) \stackrel{d}{\longrightarrow} \tau_{\leq n}(A)[1]
$$

The functors $\tau_{\leq n}$ and $\tau_{\geq n}$ are called the truncation functors. We list some of the basic properties of $\overline{\mathcal{D}}^{\leq n}, \mathcal{D}^{\geq n}$ and truncation functors:

1. $\mathcal{D}^{\leq n}$ and $\mathcal{D}^{\geq n}$ are closed under extensions;

2. $\tau_{\leq n} \circ T \simeq T \circ \tau_{\leq n+1}$;

3. If $n \geq m$, then $\tau_{\leq n} \circ \tau_{\leq m} \simeq \tau_{\leq m} \circ \tau_{\leq n} \simeq \tau_{\leq m}$, and $\tau_{\geq n} \circ \tau_{\geq m} \simeq \tau_{\geq m} \circ \tau_{\geq n} \simeq$ $\tau_{\geq n}$

4. If $m>n$, then $\tau_{\leq n} \circ \tau_{\geq m}=\tau_{\geq m} \circ \tau_{\leq n}=0$;

5. $\tau_{\geq m} \circ \tau_{\leq n} \simeq \tau_{\leq n} \circ \tau_{\geq m}$.

Define a functor

$$
H^{0}: \mathcal{D} \longrightarrow \mathcal{C}, \quad H^{0}(A)=\tau_{\leq 0}\left(\tau_{\geq 0}(A)\right)
$$

and set $H^{n}(A)=H^{0}(A[n])$. Using the truncation functors one proves the following basic result $[5,10.1 .10$.$] about the heart of a t$-structure.

Theorem 1.2 1. The heart $\mathcal{C}$ of a t-structure on $\mathcal{D}$ is an abelian category.

2. $\mathcal{C}$ is closed under extensions. 
3. If $0 \longrightarrow A \longrightarrow B \longrightarrow C \longrightarrow 0$ is an exact sequence in $\mathcal{C}$, then there exists a unique morphism $C \longrightarrow A[1]$ such that $A \longrightarrow B \longrightarrow C \longrightarrow A[1]$ is a distinguished triangle in $\mathcal{D}$.

4. $H^{0}$ is a cohomological functor. In other words, if $A \longrightarrow B \longrightarrow C \longrightarrow$ $A[1]$ is a distinguished triangle in $\mathcal{D}$, then the sequence $H^{0}(A) \longrightarrow$ $H^{0}(B) \longrightarrow H^{0}(C)$ is exact in $\mathcal{C}$.

The following lemma is stated in $[2,1.3 .19$.$] .$

Lemma 1.3 Let $\mathcal{D}^{\prime}$ be a full triangulated subcategory of $\mathcal{D}$. Let $\left(\mathcal{D}^{\leq 0}, \mathcal{D}^{\geq 0}\right)$ be a t-structure on $\mathcal{D}$. Suppose that $\mathcal{D}^{\prime}$ is stable for $\tau_{\leq 0}$ resp. $\tau_{\geq 0}$. Put

$$
\mathcal{D}^{\prime \leq 0}=\mathcal{D}^{\leq 0} \cap \mathcal{D}^{\prime} \quad \text { and } \quad \mathcal{D}^{\prime \geq 0}=\mathcal{D}^{\geq 0} \cap \mathcal{D}^{\prime}
$$

and view them as full subcategories of $\mathcal{D}$. Then the pair $\left(\mathcal{D}^{\prime \leq 0}, \mathcal{D}^{\prime \geq 0}\right)$ determines a t-structure on $\mathcal{D}^{\prime}$. Denote by $\tau_{\leq n}^{\prime}, \tau_{\geq n}^{\prime}$ the truncation functors, and by $H^{\prime n}$ cohomology functor with respect to this t-structure. If $A \in O b\left(\mathcal{D}^{\prime}\right)$, then

$$
\tau_{\leq n}^{\prime}(A)=\tau_{\leq n}(A), \quad \tau_{\geq n}^{\prime}(A)=\tau_{\geq n}(A), \quad H^{\prime n}(A)=H^{n}(A) .
$$

Hence we shall write $\tau_{\leq n}^{\prime}=\tau_{\leq n}, \tau_{\geq n}^{\prime}=\tau_{\geq n}, H^{\prime n}=H^{n}$, and we shall say the t-structure defined on $\overline{\mathcal{D}}^{\prime}$ is induce $\bar{d}$.

At the end of this section we would like to study the Grothendieck group of a triangulated category. To this end we have to introduce additional assumptions on $t$-structures. Following [2] we say that a $t$-sturcture on $\mathcal{D}$ is non-degenerate if

$$
\bigcap_{n \in \mathbb{Z}} \mathcal{D}^{\leq n}=0=\bigcap_{n \in \mathbb{Z}} \mathcal{D}^{\geq n}
$$

The importance of nondegeneracy is explained by the following result $[2,1.3 .7$.$] .$

Proposition 1.4 Suppose the t-structure on $\mathcal{D}$ is non-degenerate.

1. If $A \in O b(\mathcal{D})$, and $H^{n}(A)=0$ for all $n \in \mathbb{Z}$, then $A=0$.

2. Let $h: A \longrightarrow B$ be a morphism in $\mathcal{D}$. If $H^{n}(h): H^{n}(A) \longrightarrow H^{n}(B)$ is an isomorphism for all $n \in \mathbb{Z}$, then $h$ is also an isomorphism.

We say that a $t$-structure is bounded if for all $A \in O b(\mathcal{D})$ we have:

$$
H^{n}(A) \neq 0 \text { for at most finitely many } n \in \mathbb{Z} \text {. }
$$

Before stating the main result of this section, we give an example of a nondegenerate bounded $t$-structure. Let $D^{*}(\mathcal{A})$ be the derived category of an 
abelian category $\mathcal{A}$. Here, for $*=+($ resp. $*=b$ ) we have the derived category of complexes bounded from below (resp. bounded). Write $H^{n}(A)$ for the $n$-th cohomology group of the complex $A \in O b\left(D^{b}(\mathcal{A})\right)$. Set

$$
\begin{aligned}
& D^{\leq n}(\mathcal{A})=\left\{A \in O b\left(D^{+}(\mathcal{A})\right): H^{p}(A)=0, p>n\right\} \quad \text { and } \\
& D^{\geq n}(\mathcal{A})=\left\{A \in O b\left(D^{+}(\mathcal{A})\right): H^{p}(A)=0, p<n\right\} .
\end{aligned}
$$

This defines a non-degenerate $t$-structure on $D^{+}(\mathcal{A})$, which is called standard. The induced structure on $D^{b}(\mathcal{A})$ is non-degenerate and bounded.

Suppose that $\mathcal{T}$ is a small triangulated category. Recall that the Grothendieck group $K(\mathcal{T})$ of $\mathcal{T}$ is defined as the quotient of the free abelian group with generators $C \in O b(\mathcal{T})$, by the subgroup generated by $C-C^{\prime}-C^{\prime \prime}$, where

$$
C^{\prime} \longrightarrow C \longrightarrow C^{\prime \prime} \longrightarrow C^{\prime}[1]
$$

is a distinguished triangle in $\mathcal{T}$. Given an object $C \in O b(\mathcal{T})$, we write $[C]$ for the corresponding element in $K(\mathcal{T})$. Replacing exact triangles by exact sequences we define the Grothendieck group of an abelian category. The next theorem is a generalization of the standard fact that Euler characteristic of a bounded cochain complex depends on its cohomology. Due to a lack of adequate references we present the proof.

Theorem 1.5 Let $\mathcal{D}$ be a small triangulated category with a nondegenerate and bounded t-structure. Denote by $\mathcal{C}$ the heart of the $t$-structure. Then the inclusion $\mathcal{C} \longrightarrow D$ induces an isomorphism of Grothendieck groups $K(\mathcal{C}) \simeq$ $K(\mathcal{D})$.

Proof: Observe that by Theorem $1.2(3)$ the exact sequence in $\mathcal{C}$

$$
0 \longrightarrow C_{1} \longrightarrow C \longrightarrow C_{2} \longrightarrow 0
$$

determines a triangle in $\mathcal{D}$ :

$$
C_{1} \longrightarrow C \longrightarrow C_{2} \longrightarrow C_{1}[1] \text {. }
$$

This implies that the inclusion $\mathcal{C} \longrightarrow \mathcal{D}$ determines a homomorphism $\epsilon$ : $K(\mathcal{C}) \longrightarrow K(\mathcal{D})$. On the other hand, the long exact sequence determined by a triangle in $\mathcal{D}$ implies that the homomorphism

$$
\delta: K(\mathcal{D}) \longrightarrow K(\mathcal{C}), \quad \delta([C])=\sum_{n \in \mathbb{Z}}(-1)^{n}\left[H^{n}(C)\right]
$$

is well-defined. To show that $\epsilon$ and $\delta$ are mutually inverse, it will suffice to prove the following formula in $K(\mathcal{D})$ :

$$
[C]=\sum_{n \in Z}(-1)^{n}\left[H^{n}(C)\right]
$$


Choose $a, b \in \mathbb{Z}, a<b$ such that

$$
H^{n}(C)=0 \quad \text { if } \quad n \in \mathbb{Z} \backslash[a, b] .
$$

Consider the triangle

$$
\tau_{\leq b} C \longrightarrow C \longrightarrow \tau_{\geq b+1} C \longrightarrow \tau_{\leq b} C[1] .
$$

Using the formulas for the composition of truncations we deduce that $H^{n}\left(\tau_{\geq b+1}\right)=0$ for all $n \in \mathbb{Z}$. The non-degeneracy of the $t$-structure implies $\tau_{\geq b+1} C=0$, hence $[C]=\left[\tau_{\leq b} C\right]$. For $n \in[a, b]$ we deduce that the triangle

$$
\tau_{\leq n-1}\left(\tau_{\leq n} C\right) \longrightarrow \tau_{\leq n} C \longrightarrow \tau_{\geq n}\left(\tau_{\leq n} C\right) \longrightarrow \tau_{\leq n-1}\left(\tau_{\leq n} C\right)[1]
$$

is isomorphic to the triangle

$$
\tau_{\leq n-1} C \longrightarrow \tau_{\leq n} C \longrightarrow H^{n}(C)[-n] \longrightarrow \tau_{\leq n-1} C[1]
$$

This implies

$$
[C]=\sum_{n=a}^{b}\left[H^{n}(C)[-n]\right]+\left[\tau_{\leq a-1} C\right]=\sum_{n \in \mathbb{Z}}\left[H^{n}(C)[-n]\right]+\left[\tau_{\leq a-1} C\right] .
$$

Analogously as above we obtain $H^{n}\left(\tau_{\leq a-1} C\right)=0$ for all $n \in \mathbb{Z}$, and therefore $\left[\tau_{\leq a-1} C\right]=0$. It remains to show $[C[1]]=-[C], C \in \mathcal{D}$. This follows form the triangle

$$
C \longrightarrow 0 \longrightarrow C[1] \stackrel{-i d_{C}[1]}{\longrightarrow} C[1]
$$

\section{Equivariant sheaves}

Let $k$ be a field. Denote by $S h(X)$ the category of sheaves of $k$-vector spaces on a topological space $X$. Given a continuous map $f: X \longrightarrow Y$ we denote by

$$
f_{*}: S h(X) \longrightarrow S h(Y) \quad \text { resp. } \quad f^{*}: S h(Y) \longrightarrow S h(X)
$$

the functor of direct image resp. inverse image. Let us recall the definition of equivariant sheaves. Let $G$ be a topological group. We shall say that $X$ is a $G$-space if there exists a continuous action $G \times X \longrightarrow X,(g, x) \mapsto g x, g \in G$, $x \in X$, of $G$ on $X$. Consider the maps

$$
\begin{gathered}
e_{i}: G \times G \times X \longrightarrow G \times X, i=0,1,2, d_{i}: G \times X \longrightarrow X, i=0,1, \\
s_{0}: X \longrightarrow G \times X,
\end{gathered}
$$


defined for $g, g_{1}, g_{2} \in G, x \in X$ as follows:

$$
\begin{gathered}
e_{0}\left(g_{1}, g_{2}, x\right)=\left(g_{2}, g_{1}^{-1} x\right), e_{1}\left(g_{1}, g_{2}, x\right)=\left(g_{1} g_{2}, x\right), e_{2}\left(g_{1}, g_{2}, x\right)=\left(g_{1}, x\right), \\
d_{0}(g, x)=g^{-1} x, d_{1}(g, x)=x, s_{0}(x)=(e, x) .
\end{gathered}
$$

A $G$-equivariant sheaf is a sheaf $\mathcal{F}$ on a $G$-space $X$ together with an isomorphism of sheaves

$$
\theta_{\mathcal{F}}: d_{1}^{*} \mathcal{F} \longrightarrow d_{0}^{*} \mathcal{F}
$$

satisfying the following (cocycle) conditions:

$$
s_{0}^{*}\left(\theta_{\mathcal{F}}\right)=i d_{\mathcal{F}}, \quad e_{1}^{*}\left(\theta_{\mathcal{F}}\right)=e_{0}^{*}\left(\theta_{\mathcal{F}}\right) \circ e_{2}^{*}\left(\theta_{\mathcal{F}}\right) .
$$

We shall say that $\theta_{\mathcal{F}}$ is the $G$-structure isomorphism on $\mathcal{F}$. Suppose that $\mathcal{F}$ and $\mathcal{G}$ are $G$-equivariant sheaves with $G$-structure isomorphisms $\theta_{\mathcal{F}}$ and $\theta_{\mathcal{G}}$ respectively. We say that a morphism of sheaves $\alpha: \mathcal{F} \longrightarrow \mathcal{G}$ is a morphism of $G$-equivariant sheaves if the following diagram commutes:

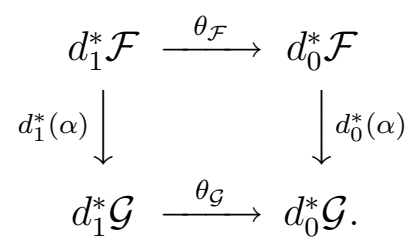

Denote by $S h_{G}(X)$ the category of equivariant sheaves on $X$. It is not difficult to show that $S h_{G}(X)$ is an abelian category. Suppose $X$ and $Y$ are $G$ spaces. A continuous map $f: X \longrightarrow Y$ is a $G$-map if it intertwines $G$-actions. The following lemma follows directly from the definition of equivariant sheaves.

Lemma 2.1 Let $f: X \longrightarrow Y$ be a G-map. Then the inverse image $f^{*}$ induces a functor

$$
f^{*}: S h_{G}(Y) \longrightarrow S h_{G}(X)
$$

The next result will play important role in our analysis of the $t$-structure on the derived category of equivariant sheaves. It holds for locally fibered maps. The special case presented below will suffice for our applications.

Lemma 2.2 Let $X$ and $M$ be $G$-spaces. Suppose $M$ is connected. Consider $P=X \times M$ as a $G$-space for the diagonal $G$-action. Let $p: P \longrightarrow X$ be the projection. Suppose $\mathcal{F} \in S h(X)$ is such that $p^{*}(\mathcal{F}) \in S h_{G}(P)$. Then $\mathcal{F} \in S h_{G}(X)$. 
Proof: The proof is based on the following simple observation. Define the full subcategory $S h(P \mid X)$ of $S h(P)$ by

$$
O b(\operatorname{Sh}(P \mid X))=\left\{\mathcal{G} \in O b(\operatorname{Sh}(P)): \mathcal{G} \simeq p^{*}(\mathcal{F})\right\}
$$

Then the functor $p^{*}: S h(X) \longrightarrow S h(P \mid X)$ provides an equivalence of categories, with inverse $p_{*}$. This follows from the formula $p_{*} p^{*}(\mathcal{F}) \simeq \mathcal{F}$.

Recall that $d_{i}, i=0,1, e_{j}, j=0,1,2, s_{0}$ are the maps appearing in the definition of equivariant sheaves on $X$. Denote by $\tilde{d}_{i}, i=0,1, \tilde{e}_{j}, j=0,1,2, \tilde{s}_{0}$ analogous maps appearing in the definition of equivariant sheaves on $P$. Let

$$
\theta_{p^{*}(\mathcal{F})}: \tilde{d}_{1}^{*}\left(p^{*}(\mathcal{F})\right) \longrightarrow \tilde{d}_{0}^{*}\left(p^{*}(\mathcal{F})\right)
$$

be the $G$-structure isomorphism on $p^{*}(\mathcal{F})$. Observe that $p \circ \tilde{d}_{i}=d_{i} \circ\left(i d_{G} \times p\right)$, $i=0,1$, hence we conclude

$$
\theta_{p^{*}(\mathcal{F})}:\left(i d_{G} \times p\right)^{*}\left(d_{1}^{*}(\mathcal{F})\right) \longrightarrow(i d \times p)^{*}\left(d_{0}^{*}(\mathcal{F})\right) .
$$

Note that $\left(i d_{G} \times p\right)^{*}: S h(G \times X) \longrightarrow S h(G \times P \mid G \times X)$ is an equivalence, hence we may define $\theta_{\mathcal{F}}=\left(i d_{G} \times p\right)^{*-1}\left(\theta_{p^{*}(\mathcal{F})}\right)$. We have to show that

$$
\theta_{\mathcal{F}}: d_{1}^{*}(\mathcal{F}) \longrightarrow d_{0}^{*}(\mathcal{F})
$$

satisfies the cocycle conditions. We start with cocycle conditions on $P$ :

$$
\tilde{s}_{0}^{*}\left(\theta_{\left.p^{*}(\mathcal{F})\right)}=i d_{p^{*}(\mathcal{F})}, \quad \tilde{e}_{1}^{*}\left(\theta_{p^{*}(\mathcal{F})}\right)=\tilde{e}_{0}^{*}\left(\theta_{p^{*}(\mathcal{F})}\right) \circ \tilde{e}_{2}^{*}\left(\theta_{p^{*}(\mathcal{F})}\right) .\right.
$$

We use the commutativity relations

$$
\left(i d_{G} \times p\right) \circ \tilde{e}_{i}=e_{i} \circ\left(i d_{G} \times i d_{G} \times p\right), \quad i=0,1,2 \text { and }\left(i d_{G} \times p\right) \circ \tilde{s}_{0}=s_{0} \circ p
$$

to rewrite the cocycle conditions on $P$ as follows

$p^{*}\left(s_{0}^{*}\left(\theta_{\mathcal{F}}\right)\right)=p^{*}\left(i d_{\mathcal{F}}\right),\left(i d_{G} \times i d_{G} \times p\right)^{*}\left(e_{1}^{*}\left(\theta_{\mathcal{F}}\right)\right)=\left(i d_{G} \times i d_{G} \times p\right)^{*}\left(e_{0}^{*}\left(\theta_{\mathcal{F}}\right) \circ e_{2}^{*}\left(\theta_{\mathcal{F}}\right)\right)$.

Finally we apply $p^{*-1}$ resp. $\left(i d_{G} \times i d_{G} \times p\right)^{*-1}$ to obtain cocycle conditions on $X$.

We say that $X$ is a free $G$-space or that $G$-action is topologically free if the following conditions are satisfied:

1. The stabilizer $G_{x}$ of any $x \in X$ is trivial.

2. The quotient map $q: X \longrightarrow X / G$ is a locally trivial fibration with fibre $G$. $[8]$.

The main property of the free actions is given by the following proposition

Proposition 2.3 Suppose $X$ is a free $G$-space and $q: X \longrightarrow \bar{X}$ is the quotient map. Then the pull-back functor defines an equivalence of categories

$$
q^{*}: S h(\bar{X}) \longrightarrow S h_{G}(X) .
$$




\section{The main result}

In this section we shall continue to assume that $G$ is a topological group. In particular, this means that $G$ is a Hausdorf topological space. Denote by $D^{*}(X)=D^{*}(S h(X)), *=+, b$ the derived category of $S h(X)$. Similarly, we shall write $D^{\leq n}(X)=D^{\leq n}(S h(X))$ and $D^{\geq n}(X)=D^{\leq n}(S h(X))$ for the full subcategories of $D^{*}(X)$ defined by the standard $t$-structure. A continuous map $f: X \longrightarrow Y$ defines the inverse image functor and the direct image functor

$$
f^{*}: D^{b}(Y) \longrightarrow D^{b}(X) \text { and } R f_{*}: D^{+}(X) \longrightarrow D^{+}(Y) \text {. }
$$

We shall say that $f: X \longrightarrow Y$ is $n$-acyclic if for any base change $Y^{\prime} \longrightarrow Y$ and the induced map $f^{\prime}: X \times_{Y} Y^{\prime} \longrightarrow Y^{\prime}$, and $\mathcal{F} \in O b\left(D^{b}\left(X \times_{Y} Y^{\prime}\right)\right)$ the natural morphism

$$
\mathcal{F}^{\prime} \longrightarrow \tau_{\leq n} R f_{*}^{\prime} f^{\prime *} \mathcal{F}^{\prime}
$$

is an isomorphism in $D^{b}\left(X \times_{Y} Y^{\prime}\right)$. We say that $f: X \longrightarrow Y$ is $\infty$-acyclic if it is $n$-acyclic for all $n \in \mathbb{Z}_{+}$. A topological space $M$ is said to be $n$-acyclic if it is connected, locally connected, and for any constant sheaf $\mathcal{V} \in S h(M)$ we have $H^{i}(M, \mathcal{V})=0$ for $i=1, \cdots, n$. In this case the natural projection $f: X=Y \times M \longrightarrow Y$ provides an example of $n$-acyclic maps $[1,1.9 .4$.].

Let $f: Y \longrightarrow X$ be a continuous map. We define the full subcategory $D^{b}(Y \mid X)$ of $D^{b}(Y)$ by the condition

$$
O b\left(D^{b}(Y \mid X)\right)=\left\{\mathcal{F} \in O b\left(D^{b}(Y)\right): \mathcal{F} \simeq f^{*}(\mathcal{G}), \mathcal{G} \in O b\left(D^{b}(X)\right)\right\} .
$$

Acyclic maps have the following important property [1, 1.9.2.].

Lemma 3.1 Suppose the map $f: X \longrightarrow Y$ is $\infty$-acyclic. Then $f^{*}$ : $D^{b}(X) \longrightarrow D^{b}(Y \mid X)$ is an equivalence of categories.

Let $X$ be a $G$-space. We say that a $G$-map $p: P \longrightarrow X$ is a resolution if the $G$-space $P$ is free. The resolution $p: P \longrightarrow X$ is $\infty$-acyclic if the map $p$ is $\infty$-acyclic.

Next we show that $\infty$-acyclic resolutions exist. Set $M=E G$, where $E G$ is the universal bundle of $G$ constructed by Milnor [6]. Then $M$ is an $\infty$-acyclic free $G$-space. Set $P=X \times M$, and consider $P$ as a $G$-space for the diagonal action of $G$. Let

$$
p: P=X \times M \longrightarrow X
$$

be the projection. It follows from Lemma 3.1 that $P$ is an $\infty$-acyclic resolution of $X$. 
Suppose that $p: P \longrightarrow X$ is a resolution of a $G$-space $X$. Denote by $q$ : $P \longrightarrow \bar{P}$ the quotient map. We recall the definition of the category $D_{G}^{b}(X, P)$. The objects of $D_{G}^{b}(X, P)$ are triples $\mathcal{F}=\left(\mathcal{F}_{X}, \overline{\mathcal{F}}, \beta_{\mathcal{F}}\right)$. Here $\mathcal{F}_{X} \in O b\left(D^{b}(X)\right)$, $\overline{\mathcal{F}} \in O b\left(D^{b}(\bar{P})\right)$, and

$$
\beta_{\mathcal{F}}: p^{*}\left(\mathcal{F}_{X}\right) \simeq q^{*}(\overline{\mathcal{F}})
$$

is an isomorphism in $D^{b}(P)$. Given objects $\mathcal{F}=\left(\mathcal{F}_{X}, \overline{\mathcal{F}}, \beta_{\mathcal{F}}\right)$ and $\mathcal{G}=$ $\left(\mathcal{G}_{X}, \overline{\mathcal{G}}, \beta_{\mathcal{G}}\right)$ in $D_{G}^{b}(X, P)$, a morphism $\alpha=\left(\alpha_{X}, \bar{\alpha}\right): \mathcal{F} \longrightarrow \mathcal{G}$ is a pair of morphisms

$$
\alpha_{X}: \mathcal{F}_{X} \longrightarrow \mathcal{G}_{X} \quad \text { resp. } \bar{\alpha}: \overline{\mathcal{F}} \longrightarrow \overline{\mathcal{G}}
$$

in $D^{b}(X)$ resp. $D^{b}(\bar{P})$ satisfying the following compatibility condition

$$
\beta_{\mathcal{G}} \cdot p^{*}\left(\alpha_{X}\right)=q^{*}(\bar{\alpha}) \cdot \beta_{\mathcal{F}}
$$

One shows $[1,2.2 .3]$ that if $R$ is another $\infty$-acyclic resolution of $X$ we have a canonical isomorphism of categories $D_{G}^{b}(X, P) \simeq D_{G}^{b}(X, R)$, hence we may define the equivariant derived category by the condition

$$
D_{G}^{b}(X)=D_{G}^{b}(X, P) .
$$

The following lemma $[1,2.3 .2]$ describes $D_{G}^{b}(X)$ as a full subcategory of complexes of sheaves in $D^{b}\left(S h_{G}(P)\right)$, which are the pull-back of complexes from $D^{b}(X)$. Its proof follows from Lemma 3.1.

Lemma 3.2 Let $p: P \longrightarrow X$ be an $\infty$-acyclic resolution of a $G$-space $X$. Set

$$
O b\left(D^{b}(\bar{P} \mid p)\right)=\left\{\mathcal{G} \in O b\left(D^{b}(\bar{P})\right): q^{*}(\mathcal{G}) \in O b\left(D^{b}(P \mid X)\right)\right\}
$$

and consider $D^{b}(\bar{P} \mid p)$ as a full subcategory of $D^{b}(\bar{P})$. Then the restriction functor

$$
\xi: D_{G}^{b}(X, P) \longrightarrow D^{b}(\bar{P} \mid p), \quad \xi(\mathcal{F})=\overline{\mathcal{F}}
$$

defines an equivalence of categories.

Next we define the structure of a triangulated $t$-category on $D_{G}^{b}(X)$. First we introduce the structure of a triangulated category and a $t$-structure on $D^{b}(\bar{P} \mid p)$.

Lemma 3.3 $D^{b}(\bar{P} \mid p)$ is an additive subcategory of $D^{b}(\bar{P})$ closed under extensions, and stable for the translation functor and the standard truncation functor $\tau_{\leq 0}$ on $D^{b}(\bar{P})$. In particular, by Lemma 1.1 and Lemma $1.3 D^{b}(\bar{P} \mid p)$ is a triangulated subcategory of $D^{b}(\bar{P})$ with induced $t$-structure. 
Proof: It is not difficult to check that $D^{b}(\bar{P} \mid p)$ is an additive subcategory stable for the translation functor. Next we show that $D^{b}(\bar{P} \mid p)$ is closed under extensions. Let

$$
\mathcal{F}_{1} \longrightarrow \mathcal{F}_{2} \longrightarrow \mathcal{F}_{3} \longrightarrow \mathcal{F}_{1}[1]
$$

be a distinguished triangle in $D^{b}(\bar{P})$, where $\mathcal{F}_{1}, \mathcal{F}_{3} \in O b\left(D^{b}(\bar{P} \mid p)\right)$. Then

$$
q^{*}\left(\mathcal{F}_{1}\right) \longrightarrow q^{*}\left(\mathcal{F}_{2}\right) \longrightarrow q^{*}\left(\mathcal{F}_{3}\right) \longrightarrow q^{*}\left(\mathcal{F}_{1}\right)[1]
$$

is a distinguished triangle in $D^{b}(P)$. Since $q^{*}\left(\mathcal{F}_{1}\right), q^{*}\left(\mathcal{F}_{3}\right) \in O b\left(D^{b}(P \mid p)\right)$, and $D^{b}(P \mid p)$ is closed under extensions $[1,1.9 .2($ iii $)]$, we conclude that $q^{*}\left(\mathcal{F}_{2}\right) \in$ $O b\left(D^{b}(P \mid p)\right)$.

It remains to show that $D^{b}(\bar{P} \mid p)$ is stable for $\tau_{\leq 0}$. Let $\overline{\mathcal{F}} \in O b\left(D^{b}(\bar{P} \mid p)\right)$. Then there exists $\mathcal{F}_{X} \in O b\left(D^{b}(X)\right)$ such that

$$
p^{*}(\overline{\mathcal{F}}) \simeq q^{*}\left(\mathcal{F}_{X}\right)
$$

Since the inverse image functors $q^{*}$ and $p^{*}$ are exact, they commute with standard truncation functors on bounded derived category of sheaves. It follows that

$$
p^{*}\left(\tau_{\leq 0} \overline{\mathcal{F}}\right) \simeq q^{*}\left(\tau_{\leq 0} \mathcal{F}_{X}\right),
$$

and we conclude that $\tau_{\leq 0} \overline{\mathcal{F}} \in O b\left(D^{b}(\bar{P} \mid p)\right)$. This completes the proof.

Now we introduce the structure of a triangulated category and a $t$-structure on $D_{G}^{b}(X)$ using Lemma 3.2 and Lemma 3.3. Explicitly, we have

$$
D_{\bar{G}}^{\leq 0}(X) \simeq D^{\leq 0}(\bar{P}) \cap D^{b}(\bar{P} \mid p), \quad D_{\bar{G}}^{\geq 0}(X) \simeq D^{\geq 0}(\bar{P}) \cap D^{b}(\bar{P} \mid p) .
$$

Denote by $\mathcal{C}$ the heart of the $t$-structure on $D_{G}^{b}(X)$, and by $S h(\bar{P} \mid p)$ the full subcategory of $S h(\bar{P})$ such that

$$
O b(S h(\bar{P} \mid p))=\left\{\overline{\mathcal{F}}: q^{*-1}(\overline{\mathcal{F}}) \in O b(S h(P \mid X))\right\} .
$$

Then the functor $\xi$ induces an equivalence of categories:

$$
\mathcal{C} \simeq S h(\bar{P}) \cap D^{b}(\bar{P} \mid p)=S h(\bar{P} \mid p) .
$$

We would like to prove that the category $\mathcal{C}$ is naturally equivalent to the category $S h_{G}(X)$. Let $\mathcal{F}_{X} \in O b\left(S h_{G}(X)\right)$. Then $p^{*}\left(\mathcal{F}_{X}\right) \in O b\left(S h_{G}(P)\right)$, hence by Proposition $2.3\left(q^{*}\right)^{-1}\left(p^{*}\left(\mathcal{F}_{X}\right)\right) \in O b\left(D^{b}(\bar{P})\right)$. Set

$$
\phi\left(\mathcal{F}_{X}\right)=\left(\mathcal{F}_{X},\left(q^{*}\right)^{-1}\left(p^{*}\left(\mathcal{F}_{X}\right)\right), i d_{p^{*}\left(\mathcal{F}_{X}\right)}\right) .
$$

Note that $\phi\left(\mathcal{F}_{X}\right) \in D_{G}^{b}(X, P) \simeq D_{G}^{b}(X)$, hence we obtain the functor

$$
\phi: S h_{G}(X) \longrightarrow D_{G}^{b}(X) .
$$


Proposition 3.4 The functor $\phi$ induces an equivalence of categories $S h_{G}(X) \simeq \mathcal{C}$. In other words, the category $S h_{G}(X)$ is naturally equivalent to the heart of the category $D_{G}^{b}(X)$.

Proof: Define $\zeta: S h_{G}(X) \longrightarrow S h(\bar{P} \mid p)$ by $\zeta\left(\mathcal{F}_{X}\right)=q^{*-1} p^{*}\left(\mathcal{F}_{X}\right)$. Let $j$ : $S h(\bar{P} \mid p) \longrightarrow D^{b}(\bar{P} \mid p)$ be the inclusion. Note that the diagram

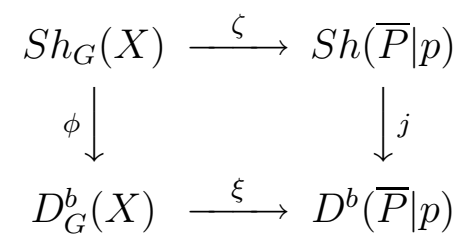

commutes, hence it will suffice to show that $\zeta$ is an isomorphism. We define the functor $\eta: S h(\bar{P} \mid p) \longrightarrow S h_{G}(X)$ as follows. Let $\overline{\mathcal{F}} \in O b(S h(\bar{P} \mid p))$. Then we can find $\mathcal{F}_{X} \in O b(S h(X))$ such that $p^{*}\left(\mathcal{F}_{X}\right) \simeq q^{*}(\overline{\mathcal{F}})$. By Lemma 2.2 $\mathcal{F}_{X} \in S h_{G}(X)$, and $p_{*} q^{*}(\overline{\mathcal{F}}) \simeq \mathcal{F}_{X}$. We set $\eta(\overline{\mathcal{F}})=p_{*} q^{*}(\overline{\mathcal{F}})$. It is not difficult to show that $\zeta \circ \eta \simeq i d_{S h(\bar{P} \mid p)}$ and $\eta \circ \zeta \simeq i d_{S h_{G}(X)}$.

Now we combine Proposition 3.4 and Proposition 1.5 to obtain the following theorem.

Theorem 3.5 The functor $\phi: S h_{G}(X) \longrightarrow D_{G}^{b}(X)$ induces an isomorphism of Grothendieck groups

$$
K\left(S h_{G}(X) \simeq K\left(D_{G}^{b}(X)\right)\right.
$$

ACKNOWLEDGEMENTS. The author was partially supported by the Ministry of Science, Education and Sport of Croatia.

\section{References}

[1] J. Bernstein and V. Lunts, Equivariant Sheaves and Functors, Lecture Notes in Mathematics 1578, Springer-Verlag, 1994.

[2] A. Beilinson, J. Bernstein and P. Deligne, Faisceaux pervers, Asterisque 100 (1982).

[3] P. Deligne, Theorie de Hodge III, Publ. Math. IHES 44 (1974), 5-78.

[4] S. Gelfand and Y. Manin, Methods of homological algebra, SpringerVerlag, Berlin, 1996.

[5] M. Kashiwara and P. Schapira, Sheaves on Manifolds, Grundlehren Math. Wiss. 292, Springer-Verlag, Berlin, 1990. 
[6] J. Milnor, Construction of universal bundles. II., Ann. of Math. (2) 63 (1956), 430-436.

[7] W. Schmid and K. Vilonen, Two geometric character formulas for reductive Lie groups, J. Amer. Math. Soc. 11 (1998), 799-867.

[8] G. Williamson, Introduction to G-equivariant sheaves, http://home.mathematik.uni-freiburg.de/geordie/EquiSheaves1.pdf

Received: June 24, 2007 